\title{
Spectra of RV Tauri stars in the Large Magellanic Cloud
}

\author{
Tom Lloyd Evans \\ School of Physics and Astronomy, University of St Andrews, North \\ Haugh, St Andrews, Fife, Scotland KY16 $9 S 5$ \\ Karen R. Pollard \\ Department of Physics and Astronomy, University of Canterbury, \\ Private Bag 4800, Christchurch, New Zealand
}

\begin{abstract}
Low-resolution ( $0.6 \mathrm{~nm})$ spectra of $24 \mathrm{RV}$ Tauri stars in the LMC shows that most are of intermediate Preston type, hence intermediate metal deficiency. Two belong to Preston's class $\mathrm{B}$, with enhanced $\mathrm{CH}$ and CN. Higher resolution spectra of the carbon star MACHO 47.2496.8 show that the ${ }^{12} \mathrm{C} /{ }^{13} \mathrm{C}$ ratio is high and that the $\mathrm{Ba}$ II lines at 455.4 and $493.4 \mathrm{~nm}$ are enhanced. This suggests that the star has undergone the third dredge-up of helium-burning products on the thermally-pulsing AGB and is now in a post-AGB stage of evolution. $J H K$ photometry from the 2MASS catalogue confirms and extends earlier findings that only moderate infrared excesses occur among this sample of RV Tauri stars.
\end{abstract}

\section{Introduction}

The RV Tauri stars represent the brightest of the Type II Cepheids and are defined photometrically by the occurrence of alternating deep and shallow minima. These stars were subdivided into three main spectroscopic groups: A (stars of near solar abundance), B (stars with weak $\mathrm{Ca}$ II lines and enhanced $\mathrm{CH}$ and $\mathrm{CN}$ bands) and C (weak CaII lines and no CN bands) (Preston et al. 1963). Most RV Tauri stars have substantial infrared excesses (Gehrz \& Woolf 1970, Gehrz 1972). These are now thought to arise from hot dust in circumstellar disks (van Winckel 1999; Lloyd Evans 1999). The Harvard survey (Gaposchkin 1970) found Type II Cepheids in the LMC and some of these have the long periods and infrared excesses typical of RV Tauri stars (Welch 1987; Laney 1991). Alcock et al. (1998) used the superb MACHO light curves to show that some of these, as well as many new examples, have the alternating deep and shallow minima of RV Tauri stars. The Harvard photographic data are not sufficiently precise to show such small differences between successive minima, so the stars in the HV sample were selected on the basis of their long periods. 


\section{Observational data}

The $J H K$ photometry of galactic RV Tauri stars is taken from Lloyd Evans (1985) and Goldsmith et al. (1987). The 2MASS all-sky survey at $J, H$ and $K$ provides photometry for the remaining stars; these have been placed on the SAAO system using the transformations given on the 2MASS website.

We have obtained spectra of all the RV Tauri stars known in the LMC at a resolution of about $0.6 \mathrm{~nm}$ over the range $360-660 \mathrm{~nm}$ using RPCS and CCD detectors on the Unit Spectrograph on the SAAO 1.9-m telescope. An attempt was made to observe the stars in the MACHO sample preferentially near minimum light, as only then are features such as CN strong, but the phases of observation are random for those stars which are known only from the earlier Harvard work. The spectra have been classified against sets of spectra of galactic standard stars. Additional spectra of the carbon star MACHO 47.2496.8 were obtained at maximum light with the RGO spectrograph on the AAT and at minimum light with the Unit Spectrograph on the 1.9-m telescope with resolutions of about $0.12 \mathrm{~nm}$ in the $450-500 \mathrm{~nm}$ region to study features relating to thermonuclear processing by the triple- $\alpha$ process and the CNO cycle.

\section{Results}

\subsection{Spectral classification}

Most stars appear to be similar to galactic RV Tauri stars of a type intermediate between groups A and C of Preston et al. (1963). This appears from the general weakness of the $\mathrm{K}$ line of $\mathrm{CaII}$ relative to expectations from the Balmer line spectral type, assuming solar chemical composition. The much later type found for $\mathrm{CN}$ than for Ca II K suggests that MACHO 82.8405.15 and 78.6698.38 belong to Preston group B. MACHO 81.8520 .15 is an example of the common stars of intermediate metallicity, while MACHO 82.8041.17 has other strong metallic features and belongs to group A. HV $12631=\mathrm{MACHO} 14.9582 .9$ has vestigial $\mathrm{TiO}$. The appearance of $\mathrm{TiO}$ is anomalous in that $\mathrm{TiO}$ would not appear in a normal giant of such early type, but this is a feature of some RV Tauri stars. Some galactic stars of group A, such as UMon, show much stronger $\mathrm{TiO}$ at times. Hydrogen emission, at least at $\mathrm{H} \alpha$, is common and, given the phasing difficulties, it is likely that all stars show it at times. The emission is most pronounced on the rising branch of the light curve.

\subsection{The Carbon Star MACHO 47.2496.8}

MACHO 47.2496.8 is a carbon star (Pollard \& Lloyd Evans 1999). This star is unique among known RV Tauri stars, so two spectra were taken at higher resolution to check on diagnostic features of carbon stars. The star was observed with the AAT near maximum light (phase 0.71 , where the deep minimum occurs at phase 0.00) on JD 2451114.12 and at SAAO near minimum light (phase 0.99), on JD 245 1145.55. The bands are almost invisible on the first spectrum but strong on the second. The AAT spectrum shows enhanced BaII at 455.4 and $493.4 \mathrm{~nm}$, while the SAAO spectrum shows enhanced Ba II at $493.4 \mathrm{~nm}$ relative to typical $\mathrm{R}$ stars with similar $\mathrm{C}_{2}$ bands but comparable to that in $\mathrm{CH}$ stars. 


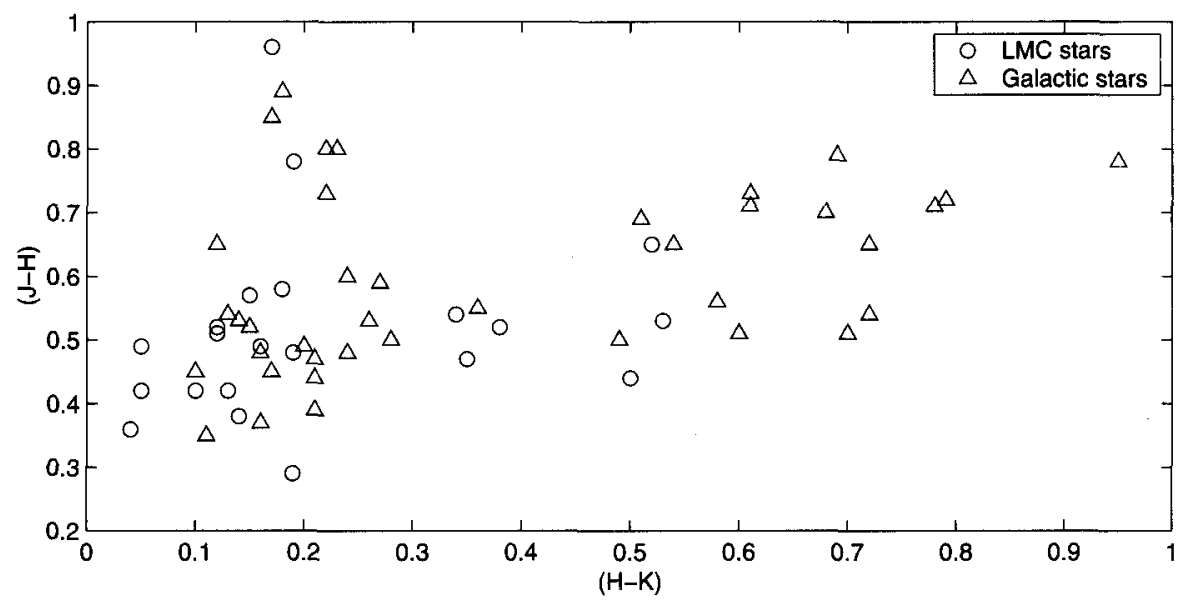

Figure 1. The $(J-H)-(H-K)$ diagram for galactic and LMC RV Tauri stars.

The $474.4 \mathrm{~nm}$ bandhead of ${ }^{13} \mathrm{C}$ is very much weaker than in the hot $\mathrm{J}(\mathrm{R})$ stars in the field or the $\mathrm{CH}$ stars in $\omega$ Centauri, which have similar total bandstrength, so the ${ }^{12} \mathrm{C} /{ }^{13} \mathrm{C}$ ratio must be quite large. Both these features suggest that the star has been enriched in ${ }^{12} \mathrm{C}$ and s-process elements by triple- $\alpha$ processing and the subsequent third dredge-up on the AGB.

\subsection{Photometry}

The positions of these RV Tauri stars in the $(J-H)-(H-K)$ diagram have been compared with galactic examples (Fig. 1). The LMC stars belong with those galactic stars which have no, or only moderate, excesses at $K$. This is similar to what Welch (1987) and Laney (1991) found for smaller samples of stars chosen from the original HV sample of Gaposchkin (1970), and is in accordance with expectations for stars belonging to a moderately metal-weak population.

Laney (1991) found that HV 5756 = MACHO 78.6338.24 and HV $12631=$ MACHO 14.9582.9 are unusually red in $(J-H)$. The 2MASS data suggest that HV 5756 owes its unusual colour in the SAAO data to a nearby companion; the 2MASS data and that of Welch (1987) do not show any peculiarity of colour for this star. The 2MASS data confirm the red $(J-H)$ of HV $12631=$ MACHO 14.9582.9. It is of interest that this star was the only one for which the low-resolution spectra hinted at the presence of $\mathrm{TiO}$ bands in the red.

\section{Discussion}

Most of the stars in our sample fulfil the main photometric and spectroscopic criteria for membership in the RV Tauri class and are probably similar to galactic RV Tauri stars, which in most cases have dusty disks and are likely to be members of binary systems (van Winckel 1999; Lloyd Evans 1999). These stars show no sign of enhanced carbon, other than the strong CN in stars of Preston's B 
group, which are generally believed to owe their peculiarities to extreme selective depletion of refractory elements in the dusty disk (Giridhar, Rao \& Lambert 1994). There is also no universal enhancement of s-process elements, in the case of galactic stars observed at high resolution, so the RV Tauri stars in general need not be in a classical post-AGB stage of evolution. They may instead have evolved prematurely off the AGB or even the RGB as a result of mass loss stimulated by the presence of a companion (Giridhar, Lambert \& Gonzalez 2000). This is supported by the low ${ }^{12} \mathrm{C} /{ }^{13} \mathrm{C}$ values found for several RV Tauri stars. Such low values are routinely found in old, low-mass stars on the red giant branch (RGB), whereas stars which have evolved through the thermally-pulsing AGB stage of evolution have been enriched with ${ }^{12} \mathrm{C}$ produced by the triple-alpha process and generally have a high ${ }^{12} \mathrm{C} /{ }^{13} \mathrm{C}$ ratio, as Lambert et al. (1986) found to be true of most cool carbon stars.

The carbon-rich MACHO 47.2496.8 is the one striking exception to this scenario. Evidence presented in Section 3.2 suggests that it is a post-AGB star which has passed through the thermal-pulsing, third dredge-up stage of evolution on the AGB. It follows that the Cepheid instability strip may be populated by two sets of RV Tauri stars with very different evolutionary histories.

Acknowledgments. This publication makes use of data from the Two Micron All Sky Survey, a joint project of the University of Massachusetts and the Image Processing and Analysis Center/California Institute of Technology, funded by NASA and the NSF.

\section{References}

Alcock, C. et al. 1998, AJ, 115, 1921

Gaposchkin, S. 1970, Smithsonian Ap. Obs. Special Report 310

Gehrz, R.D. 1972, ApJ, 178, 715

Gehrz, R.D. \& Woolf, N.J. 1970, ApJ, 161, L213

Gilroy, K.K. 1989, ApJ, 347, 835

Giridhar, S., Lambert, D.L. \& Gonzalez, G. 2000, ApJ, 531, 521

Giridhar, S., Rao, N.K. \& Lambert, D.L. 1994, ApJ, 437, 476

Goldsmith, M.J. et al. 1987, MNRAS, 227, 143

Lambert, D.L. et al. 1986, ApJS, 62, 373

Laney, C.D. 1991, in IAU Symp. 148, The Magellanic Clouds, ed. R. Haynes \& D. Milne (Dordrecht: Reidel), 109

Lloyd Evans, T. 1985, MNRAS, 217, 493

Lloyd Evans, T. 1999, in IAU Symp. 191, AGB Stars, ed. T. Le Bertre, A. Lebre \& C. Waelkens (San Francisco: ASP Publications), 453

Pollard, K.R. \& Lloyd Evans, T. 2000, AJ, 120, 3098

Preston, G.W., Krzeminski, W., Smak, J. \& Williams, J.A. 1963, ApJ, 137, 401 Shetrone, M.D. 2003, ApJ, 585, L45

van Winckel, H. 1999, in IAU Symp. 191, AGB Stars, ed. T. Le Bertre, A. Lebre \& C. Waelkens, 465

Welch, D.L. 1987, ApJ, 317, 672 